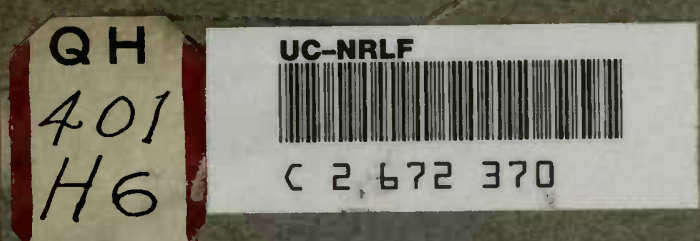



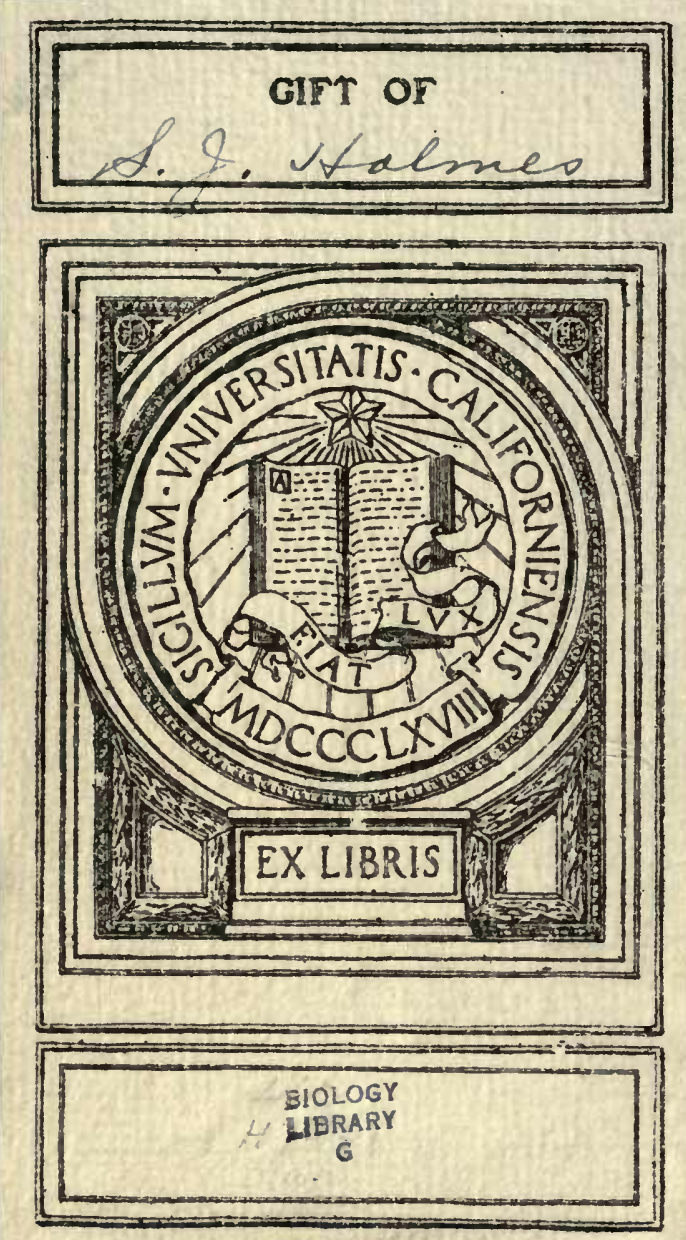
[Reprinted from ScIence, N. S., Vol. XLII., No. 1079, Pages 300-303, September 3, 1915]

\section{ARE RECESSIVE CHARACTERS DUE TO LOSS?}

Since the presence-absence theory came into vogue it has become quite customary to regard recessive characters as due to the absence of something in the germ plasm on which the corresponding dominant character depends. The nomenclature of the presence-absence theory has been adopted by most writers on Mendelian inheritance, and it has afforded a useful and convenient method of expressing gametic formulas, although, as Morgan has shown, there are cases in which it leads to inconsistent results. While it is often recognized that this nomenclature is a purely symbolic scheme of indicating how certain characters behave in inheritance, the habitual employment of the system in the search for formulas which will designate by a series of large and small letters the gametic constitution of the organisms one is dealing with, has a strong tendency to influence one's views in regard to several important problems of heredity and evolution. I can not but think that the opinions of many students of genetics have been unduly influenced by their formulas. Formulas are excellent servants but bad masters. Almost involuntarily a certain interpretation is attached to their symbolism which is apt to have the practical effect of actual belief if it does not succeed in producing it.

Since the establishment of Mendel's law and its successful employment in elucidating many previously enigmatical phenomena of inheritance, heritable variations have commonly come to be considered as due to the addition or subtraction of discrete units of germ plasm, the bearers of unit characters. Professor Bateson in his "Problems of Genetics" says in regard to substantive variations that

we are beginning to know in what such variations consist. These changes must occur either by the addition or loss of factors.
And further on he makes the following significant statement:

Recognition of the distinction between dominant and recessive characters has, it must be conceded, created a very serious obstacle in the way of any rational and concrete theory of evolution. While variations of all kinds could be regarded as manifestations of some mysterious instability of organisms this difficulty did not occur to the minds of evolutionists. To most of those who have taken part in genetic analysis it has become a permanent and continual obsession. With regard to the origin of recessive variations, there is, as we have seen, no special difficulty. They are negative and are due to absences, but as soon as it is understood that dominants are caused by an addition we are completely at a loss to account for their origin, for we can not surmise any source from which they have been derived.

In his more recent address before the British Association, Bateson not only interprets all recessive characters as due to loss, but suggests that dominant characters may have arisen by the removial of inhibiting factors, thereby causing a "release" of the characters which previously lay latent in the germ plasm, and producing the appearance (but only the appearance) of new variations. He says:

In spite of seeming perversity we have to admit that there is no evolutionary change which in the present state of our knowledge we can positively declare to be not due to loss.

If we explain not only the actual disappearance of characters as caused by germinal loss, but the appearance of new characters as due to the loss of inhibitors which prevented these characters from manifesting themselves, it is theoretically possible to consider the whole process of progressive evolution as accomplished by the sloughing off of inhibiting factors. Such a doctrine which naturally reminds one of the extravagancies of the theory 
of emboitement might have proved quite acceptable to Leibnitz, Haller, or Bonnet, but, unless I misunderstand him, Professor Bateson has presented this view more as an illustration of the bankruptcy of present evolutionary theory than as a matter of serious conviction of his own. I will not discuss this interesting speculation further than to observe that any interpretation of variation which logically leads to such a standpoint naturally incurs a very justifiable suspicion of unsoundness. It may be that in the case of any particular variation we are unable to positively declare that it is not due to loss, but on the other hand we are unable to positively declare that most variations are due to loss. I think I am not going too far in stating that a germinal variation due to loss has not been proved to occur in any single case. If it is legitimate to explain the appearance of new characters as due to the removal of inhibitors, we may also explain the apparent loss of a character as due to the advent of inhibitors. It is surely justifiable to assume that inhibitors can come into an organism somehow if we are permitted to make such frequent use of their disappearance in accounting for the origin of new variations. The plain fact is that we know practically nothing of the changes in the germ plasm which we postu-

- late as the causes of variability. It is easy to assume the existence of an inhibitor to bring any particular variation into line with one's general theory, but such explanations are purely formal and therefore of little scientific value.

While few would be inclined to follow Bateson in his rather paradoxical interpretation of dominance, the doctrine that recessiveness is due to loss is coming to be quite prevalent among workers in genetics. One of the chief reasons for regarding so much of the variation that has arisen among domestic animals as caused by the loss of factors is the fact that the crossing of different varieties often produces a reversion toward the ancestral type. If we regard the ancestor of our races of domestic mice for instance as possessing a full complement of factors, and assume that the different varieties have arisen by the dropping out of one or more factors in this variety, and one or more other factors in that variety, then when these varieties are crossed the hybrid may possess all the factors of the original ancestor and hence show a reversion to type. On the basis of this assumption one can make out gametic formulas for the different varieties of a species, test them by breeding experiments, and thus verify their correctness. Gametic fomulas obtained in this way doubtless symbolize a truth in regard to the germinal constitution of the organisms in question. The value of such formulas is no longer a matter of doubt, and is quite independent of the various interpretations that can be made concerning the nature of the symbolism, just as chemical formulæ are of value quite irrespective of the various theories of the constitution of atoms.

Consider the origin of a black mouse according to the presence-absence hypothesis. We may explain the origin of a black mouse by saying that it is caused by the absence of the agouti or ticking factor that breaks up the color of the hair into bars. Gray is therefore black plus an agouti factor. But does it follow that because we can interpret the facts in this way, and interpret them consistently so far as breeding experiments are concerned, the change that has taken place in the germ plasm that produced a black mouse was really a loss? Such a change is frequently assumed to be the result of an actual loss of a little discrete unit of some sort in the germ plasm. De Vries has interpreted recessiveness as due to the latency or loss of potency of pangens, but we may also assume that the germinal basis of the character in question has undergone a change of such a character that without becoming inactive it ceases to function in its usual way. The agouti factor (commonly designated by $G$ ) may be regarded as dependent on a part of the germ plasm, a section of a chromosome possibly, which when present causes the barring of color in the hair. When a black mouse arises we may suppose that something takes place in G. It is not necessarily a change in the direction of either chemical or organic simplicity any more than it is necessarily a loss of substance. The fact

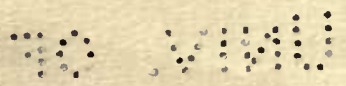


that the modified condition is recessive to $G$ proves nothing whatsoever in regard to the nature of the transformation that has occurred in the germ plasm. Assuming that $\mathrm{G}$ is not actually lost, but modified into another kind of substance $\mathrm{g}$, the recessiveness of $\mathrm{g}$ may be due to the fact that its activity is manifested in a different way, relative slowness of its metabolism, or to various other conceivable causes.

There is, I believe, no good reason for considering the recessiveness of a character as due to the relative simplicity of its germinal basis. Many variations of a minus character are recessive, but there are numerous exceptions to this rule, as is illustrated by the dominance of the hornless condition in cattle, the short tail of Manx cats, and the lack of beards in certain kinds of wheat. Suppose we have two allelomorphic units (assuming for the present that there are such things as germinal units) $\mathrm{A}$ and $\mathrm{A}^{\prime}$, one of which tends to produce a relatively simple development of a part and the other a relatively complex development of a particular part of the body. The one A calls forth, say, simple horns, the other branched horns. $\mathrm{A}$ and $\mathrm{A}^{\prime}$ presumably differ chemically, and the development of the part in question depends not upon $\mathbf{A}$ or $\mathbf{A}^{\prime}$ alone, but upon how these agencies affect other parts of the body during development. Will the simpler substance or organic unit call forth the simpler structure in the adult body? Inasmuch as the development of any organ involves activities in which a larger number of elements are concerned it seems not at all improbable that the simpler substance or unit might conspire to produce the more complex organ. Now suppose that the forked horn proved to be dominant over the simple horn. What conclusions would we be entitled to draw from this fact concerning the germinal basis of these characters? Obviously none.

Whether we interpret a variation as a gain or a loss is in most cases a purely arbitrary matter. In sugar corn there is a loss of starch but there is a gain of sugar. Does sugar corn therefore represent a plus or a minus variation? Consider the familiar cases of rose comb and pea comb in poultry. Both of these variations are dominant over the primitive condition of single comb. Yet both breeds carry the basis for the production of single comb in their germ plasm. It is commonly assumed that both conditions represent single comb plus something. We may suppose that in a certain chromosome a change has taken place which results in the development of rose comb. This change, for all that we know, may be due to the loss or impairment of a portion of germ plasm, or it may be due to a change not properly describable as either a gain or loss. We may regard rose and pea comb as more or less pathological deviations based on germinal defect, as true progressive variations, or simply as normal variations neither progressive nor retrogressive. So far as complexity of structure is concerned it may be a matter of dispute whether rose comb, pea comb, or single comb represents the higher grade of development.

But, it may be asked, are not color varieties commonly due to loss, and is not this obviously the case with albinism? In many varieties there has certainly been a loss of pigment, but has there been a dropping out of factors? It by no means follows. The factors represented by small letters in our color formulas are by no means missing entities. They are changed so that they occasion a diminished production of certain pigments, but in other respects they may be as potent as before. The albino does not produce pigment, but there may be other substances in the place of pigment that would distinguish the albino as a positive variation when judged by other standards. The animals whose gametic formulas contain a number of small letters are not necessarily more imperfect or perhaps I should say incomplete than their congeners which carry a large number of dominant characters.

Of course there may be varieties due to losses of germinal material. Considering the complex mechanism of mitosis, and the opportunities afforded for the loss of chromatin during this process, such variations are not improbable a priori. But there is not the slightest warrant in the fact of recessiveness per se for the doctrine that all recessive variations are produced by this method. The origin 
of so-called unit characters may depend, for the most part, not upon germinal loss or gain, but simply on transformation. Viewed in this simple and natural way the appearance of a new dominant character is not an event to be marvelled at. Dominant and recessive characters not improbably owe their origin to much the same causes. At least we do not know that they do not. Concerning the real causes of variations of any kind we know very little more than we did when Darwin commented on our profound ignorance of this subject. It is therefore premature to pin our faith to any particular theory of the origin of variation and especially to draw far-reaching conclusions regarding evolution on the basis of such an interpretation. We may conceive variability as due to germinal losses or gains for the sake of our formulas, and there may be little harm in so doing so long as it is clearly realized that the procedure is a purely arbitrary and schematic method of recording certain facts of inheritance. But when we make the serious attempt to apply the conception to what actually takes place in the germ plasm we encounter a fruitful source of fallacies.

\section{S. J. Holmes}

UNIVERSITY OF CALIFORNIA 


\section{3}

\section{Hrisio:}

BrOLOCy

UNIVERSITY OF CALIFORNIA LIBRARY

THIS BOOK IS DUE ON THE LAST DATE STAMPED BELOW

AN INITIAL FINE OF 25 CENTS WILL BE ASSESSED FOR FAILURE TO RETURN THIS BOOK ON THE DATE DUE. THE PENALTY WILL INCREASE TO 50 CENTS ON THE FOURTH DAY AND TO $\$ 1.00$ ON THE SEVENTH DAY

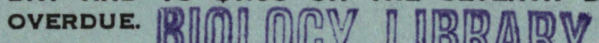

\begin{tabular}{|c|c|}
\hline NOV 151938 \\
\hline NOV 91940 \\
\hline
\end{tabular}


